\title{
Mathematical analysis and stability of a chemotaxis model with logistic term
}

\author{
J. Ignacio Tello
}

\begin{abstract}
SUMMARY
In this paper we study a non-linear system of differential equations arising in chemotaxis. The system consists of a PDE that describes the evolution of a population and an ODE which models the concentration of a chemical substance. We study the number of steady states under suitable assumptions, the existence of one global solution to the evolution problem in terms of weak solutions and the stability of the steady states.
\end{abstract}

KEY WORDS: chemotaxis; stability of stationary solutions; parabolic equations; reinforced random walks

\section{INTRODUCTION}

Chemotaxis is the ability of microorganisms to respond to chemical signals by moving along the gradient of the chemical substance, either toward the higher concentration (positive taxis) or away from it (negative taxis).

Over the last few decades a rich variety of mathematical models for studying chemotaxis has appeared. One of the first was presented by Keller and Segel [1,2]. It describes the density distribution of a type of bacteria 'Dyctyostelium discoideum' (denoted by $p$ ) and a chemical concentration, $w$, in a coupled system of partial differential equations

$$
\begin{aligned}
\frac{\partial p}{\partial t} & =\Delta p-\operatorname{div}(p \chi(w) \nabla w) \\
0 & =\Delta w+(p-1)
\end{aligned}
$$

Contract/grant sponsor: DGES (Spain); contract/grant number: REN 2000/0766

Contract/grant sponsor: European Union; contract/grant number: HPRN-CT-2002-00274 
After this study, there has been great interest in the analysis of similar models (see References [3-7] and reference there).

In the last fifteen years another model, called reinforced random walks, has been developed to understand the mechanism of chemotaxis (see References [8,9] and reference there). Chemotaxis also appears in many other phenomena, such as for instance in the formation of capillary blood by endothelial cells. Recently, Anderson and Chaplain [10] and Levine et al. [11] have introduced several models for angiogenesis. These authors study the growth of tumors based on the analysis of the relevant biochemical processes and the methodology of reinforced random walks.

Friedman and Tello [5] study the models proposed in Levine and Sleeman [9] and Othmer and Stevens [8] under suitable conditions in chemotactic coefficient and production terms.

Fontelos et al. [4] study the model proposed in Reference [11]. This system of equations does not have a logistic growth term and non-constant steady states appear (see Reference [4]). Fontelos et al. [4] prove the existence of global solutions in the space $C_{x, t}^{2+\beta, 1+1 / \beta}$ and analyse the asymptotic behaviour of the solutions and their stability. They consider that the production of the chemical substance depends on $p$ and $x$. Therefore the production term is non-decreasing in $w$ (essential assumption in the proof of the results of this paper).

In this paper we consider the system

$$
\begin{gathered}
\frac{\partial p}{\partial t}=\operatorname{div}(d \nabla p-p \chi(w) \nabla w)+r p(N-p) \quad x \in \Omega t>0 \\
\frac{\partial w}{\partial t}=h(p, w) \quad x \in \Omega \quad t>0
\end{gathered}
$$

where $d$ is the diffusion constant, $\chi(w)$ is the chemotactic sensitivity and $r, N$ are positive constants. $h(p, w)$ represents the production of the chemical substance by the living organisms. Depending on the process, $\chi(w)$ and $h(p, w)$ can take different forms. The boundary conditions for $p$ are

$$
\frac{\partial p}{\partial n}-p \chi(w) \frac{\partial w}{\partial n}=0 \quad x \in \partial \Omega \quad t>0
$$

where $\partial p / \partial n$ is the outward normal derivative and initial conditions are

$$
p(x, 0)=p_{0}(x), \quad w(x, 0)=w_{0}(x) \quad x \in \Omega
$$

As in Reference [12] we consider the logistic growth term $r p(N-p)$ in the equation which models the density of the population.

Myerscough et al. [12] performed a numerical study of the steady states in case that $w$ satisfies an elliptic equation of the type

$$
-\Delta w=h(p, w) \quad x \in \Omega
$$

They focus on the role of boundary conditions and find spatially non-constant solutions for different boundary conditions and some non-linear functions $h$. 
We assume throughout the paper that the positive constants $\bar{q}, \bar{p}, \bar{w}$ exist and satisfy

$$
\begin{gathered}
\bar{q}>\max _{x \in \Omega}\left\{p_{0}(x), N\right\}, \quad \bar{w}>\max _{x \in \Omega}\left\{w_{0}(x)\right\}, \quad \bar{p}=\bar{q} \exp \left\{\int_{0}^{\bar{w}} \chi(w) \mathrm{d} w\right\} \\
h(\bar{p}, \bar{w})=0 \quad h(0,0)=0
\end{gathered}
$$

$\chi$ and $h$ satisfy

$$
\begin{gathered}
\chi, h \text { belong to } C^{2}, \text { for } 0 \leqslant p \leqslant \bar{p}, 0 \leqslant w \leqslant \bar{w} \\
\frac{\partial h}{\partial p}>0 \quad \text { if } 0 \leqslant p \leqslant \bar{p}, \quad 0 \leqslant w \leqslant \bar{w} \\
p \chi \frac{\partial h}{\partial p}+\frac{\partial h}{\partial w}<0 \text { if } 0 \leqslant p \leqslant \bar{p}, \quad 0 \leqslant w \leqslant \bar{w} \\
\chi(w)>0, \quad \text { for } 0 \leqslant w \leqslant \bar{w}
\end{gathered}
$$

$\Omega \subset \mathbb{R}^{n} \quad(n \leqslant 3)$ is an open and bounded domain with $\partial \Omega \in C^{2+\beta}$

Assumption (10) means that the organisms move toward the higher concentration of the chemical substance.

We also assume that the initial data satisfy

$$
\begin{aligned}
& 0 \leqslant p_{0}(x) \in H^{1}(\Omega) \cap L^{\infty}(\Omega) \quad 0 \leqslant w_{0}(x) \in H^{2}(\Omega) \quad \text { and } \\
& \frac{\partial p_{0}}{\partial n}=\frac{\partial w_{0}}{\partial n}=0 \quad \text { on } \partial \Omega
\end{aligned}
$$

Assumptions (7)-(10) are satisfied, for instance,

$$
\begin{gathered}
h(p, w)=\mu p-w \quad \chi=\text { constant } \\
h(p, w)=\mu p-\frac{w}{\alpha+w} \quad \chi=\frac{\beta+w}{\alpha+w} \\
h(p, w)=\mu p \frac{w}{\alpha+w}-v w \quad \chi=\frac{\gamma}{\beta+w}
\end{gathered}
$$

for a range of parameters and some initial data. Several researchers in this area are particularly interested in the case $(15)$ where $\chi(w)$ appears in the literature in the form $\ln (\phi(w))^{\prime}$ for $\phi(w)=(\beta+w)^{\gamma}$. In case (15) assumptions (5)-(9) hold and Theorems 1.1-1.3 can be applied if $\bar{p}$ satisfies $N<\bar{p}<v \beta \alpha / \gamma \bar{w}$ where $\bar{p}$ and $\bar{w}$ are defined by (5), (6).

Solutions to (2)-(4) which are biologically meaningful must satisfy

$$
0 \leqslant p(x, t)<\infty, \quad 0 \leqslant w(x, t)<\infty
$$

Set $\Omega_{T}=\Omega \times(0, T)(0<T<\infty)$. We will assume throughout the paper that $d=1$. 
The main results of this work are the following theorems:

Theorem 1.1

Under the assumptions (7)-(11), the steady states $\left(p_{*}, w_{*}\right)$ of $(2)-(4)$ satisfying $(16)$ are constant and given by

$$
(0,0) \text { or }(N, \Psi(N))
$$

where $\Psi(N)$ is the unique solution to $h(N, \Psi(N))=0$.

Theorem 1.2

Under the assumptions (7)-(11), there exists a unique global solution $(p, w)$ to $(2)-(4)$ satisfying

$$
p \in L^{2}\left(0, T: H^{2}(\Omega)\right) \cap L^{\infty}\left(\Omega_{T}\right) \quad w \in L^{\infty}\left(0, T: H^{2}(\Omega)\right) \cap L^{\infty}\left(\Omega_{T}\right)
$$

for any $T<\infty$.

Theorem 1.3

If the initial values $\left(p_{0}, w_{0}\right)$ satisfy

$$
\sup _{x \in \Omega}\left|p_{0}(x)-N\right|+\sup _{x \in \Omega}\left|w_{0}(x)-\Psi(N)\right| \leqslant \varepsilon
$$

where $\varepsilon$ is small enough and (7)-(11) hold. Then, the solution $(p, w)$ to $(2)-(4)$ has the asymptotic behaviour

$$
p \rightarrow N, \quad w \rightarrow \Psi(N) \quad \text { when } t \rightarrow \infty \quad \text { in } L^{s}(\Omega)
$$

for any $s \leqslant \infty$ if $n=1, s<\infty$ if $n=2$ and $s<6$ if $n=3$.

Assumptions (8) and (9) define the behaviour of the solution. Following Levine and Sleeman [9] Equations (2)-(4) can be considered in the 'Hodograph plane': applying the implicit function theorem to Equation (1) and as a result of (8) and (9) we obtain

$$
p=\psi\left(w_{t}, w\right) \quad \frac{\partial \psi}{\partial w_{t}}=\left(\frac{\partial h}{\partial p}\right)^{-1} \quad \frac{\partial \psi}{\partial w}=-\frac{\partial h}{\partial w}\left(\frac{\partial h}{\partial p}\right)^{-1}
$$

If $\Omega=(0, L) \subset \mathbb{R}$, substituting it in (2) it results

$$
\begin{aligned}
& \psi_{w_{t}} w_{t i}+\psi_{w} w_{t}-\left(\psi_{w_{t}} w_{x x t}+\psi_{w} w_{x x}+2 \psi_{w_{t} w_{x}} w_{x} w_{x t}\right) \\
& +\chi \psi w_{x x}+\chi \psi_{w_{t}} w_{x t} w_{x}+\left(\chi \psi \psi_{w}+\chi^{\prime} \psi\right)\left(w_{x}\right)^{2}=r \psi(N-\psi)
\end{aligned}
$$

Consider now the second order operator

$$
\mathscr{L}(w):=w_{t t} \psi_{w_{t}}+\left(-\psi_{w}+\chi \psi\right) w_{x x}+2 b w_{x t}
$$


where $b=\frac{1}{2}\left(-2 \psi_{w w} w_{x}+\chi \psi_{w_{t}} w_{x}\right)$. Then Equations (2), (1) become

$$
\mathscr{L}(w)=k\left(w, w_{x}, w_{t}, w_{x x t}, \psi, \psi_{w}, \psi_{w_{t}}, \psi_{w w_{t}}\right)
$$

Since the discriminant

$$
b^{2}-\psi_{w_{t}}\left(-\psi_{w}+\chi \psi\right)=b^{2}-\left[\frac{\partial h}{\partial p}\right]^{-1}\left(\frac{\partial h}{\partial w}\left(\frac{\partial h}{\partial p}\right)^{-1}+\chi \psi\right)=b^{2}-\left[\frac{\partial h}{\partial p}\right]^{-2}\left(\frac{\partial h}{\partial w}+\chi \psi \frac{\partial h}{\partial p}\right)
$$

is strictly positive (by (9)) $\mathscr{L}$ is clearly an hyperbolic operator. Assumption (9) implies that $h$ is strictly increasing in $w$. If $(9)$ is substituted by $p \chi(\partial h / \partial p)+\partial h / \partial w>0$, there are no control on the type of the differential operator $\mathscr{L}$, and it could be parabolic, elliptic or hyperbolic. Then the arguments used to prove Lemma 3.2 cannot be applied and blow-up could occur (as in case $r=0$ for some values of the parameters, see Reference [9] for details).

\section{ON THE STEADY STATES}

\subsection{Proof of Theorem 1.1}

Let us consider the solutions to the stationary problem

$$
\begin{gathered}
\operatorname{div}(\nabla p-p \chi(w) \nabla w)+r p(N-p)=0 \quad x \in \Omega \\
h(p, w)=0 \quad x \in \Omega
\end{gathered}
$$

Since $h_{p}>0, h_{w}<0$ (by (8) and (9)), we can apply the implicit function theorem to solve Equation (20) in the form $p=\Psi(w)$ and obtain

$$
\Psi^{\prime}(w)=-\frac{h_{w}}{h_{p}}>0 \quad \text { and } \quad \Psi(0)=0
$$

Let $\left(p_{*}, w_{*}\right)$ be a stationary solution, then $h\left(p_{*}, w_{*}\right)=0$ and $p_{*}=\Psi\left(w_{*}\right)$. Substituting this in (2) we get

$$
-\operatorname{div}\left\{\frac{1}{h_{p}}\left(h_{w}+p_{*} \chi\left(w_{*}\right) h_{p}\right) \nabla w_{*}\right\}+r \Psi\left(w_{*}\right)\left(N-\Psi\left(w_{*}\right)\right)=0 \quad \text { in } \Omega
$$

with boundary conditions

$$
\Psi^{\prime}\left(w_{*}\right) \frac{\partial w_{*}}{\partial n}-p_{*} \chi\left(w_{*}\right) \frac{\partial w_{*}}{\partial n}=\frac{-1}{h_{p}}\left(h_{w}+p_{*} \chi\left(w_{*}\right) h_{p}\right) \frac{\partial w_{*}}{\partial n}=0
$$

i.e.

$$
\frac{\partial w_{*}}{\partial n}=0 \quad x \in \partial \Omega
$$


Lemma 2.1

Any solution $w_{*}$ to (23), (24) satisfying (16) belongs to $C^{1}(\Omega)$.

Proof

Let us consider the function $\Phi$ defined by

$$
\Phi\left(w_{*}\right)=\int_{0}^{w_{*}} \frac{1}{h_{p}(\Psi(s), s)}\left(h_{w}(\Psi(s), s)+\Psi(s) \chi(s) h_{p}(\Psi(s), s)\right) \mathrm{d} s
$$

By (7)-(9) we know that $\Phi \in C^{1}$ and $\Phi^{\prime}<0$. Substituting this into (23) we get (assuming (16))

$$
-\Delta \Phi\left(w_{*}\right) \in L^{\infty}(\Omega)
$$

and then $\Phi\left(w_{*}\right) \in W^{2, \infty}(\Omega) \subset C^{1}(\Omega)$. By regularity of $\Phi$ we get the desired result. Extra regularity can be obtained if $\chi$ and $h$ have additional regularity.

Let us consider the positive part function defined by

$$
(s)_{+}=\left\{\begin{array}{cc}
s & \text { if } s>0 \\
0 & \text { otherwise }
\end{array}\right.
$$

Taking $\left(N-\Psi\left(w_{*}\right)\right)_{+}$as test function in (23), we obtain

$$
\int_{\Omega} \frac{1}{h_{p}}\left(h_{w}+p_{*} \chi\left(w_{*}\right) h_{p}\right) \frac{1}{-\Psi^{\prime}}\left[\nabla\left(N-\Psi\left(w_{*}\right)\right)_{+}\right]^{2} \mathrm{~d} \sigma+\int_{\Omega} r \Psi\left(w_{*}\right)\left(N-\Psi\left(w_{*}\right)\right)_{+}^{2} \mathrm{~d} \sigma=0
$$

By (22), (8) and (9) we get

$$
\int_{\Omega} r \Psi\left(w_{*}\right)\left(N-\Psi\left(w_{*}\right)\right)_{+}^{2} \mathrm{~d} \sigma=0
$$

hence, by (22) and (16), $w_{*}$ satisfies

$$
\Psi\left(w_{*}\right)=0 \quad \text { or } \quad \Psi\left(w_{*}\right) \geqslant N
$$

Integrating (23), (24) we get

$$
\int_{\Omega} \Psi\left(w_{*}\right)\left(N-\Psi\left(w_{*}\right)\right) \mathrm{d} \sigma=0
$$

By Lemma 2.1, continuity of $\Psi$ and (25), we deduce the desired result.

2.2. On infinitely many steady states (a simple example)

If assumption (9) is not satisfied then infinitely many solutions to (20), (21) can occur. 
Let us consider a simple case where $\chi$ is a positive constant and $h(p, w)=\mu p-w$ for $\mu \chi N / 2=1$. Then $w=\mu p$, and $p$ satisfies

$$
\begin{cases}-\operatorname{div}\{\nabla p-\chi \mu p \nabla p\}=r p(N-p) & x \in \Omega \\ \frac{\partial p}{\partial n}=0 & x \in \partial \Omega\end{cases}
$$

Let us define $u=p-(1 / N) p^{2}$ which satisfies the well known problem

$$
\begin{cases}-\Delta u=r N u & x \in \Omega \\ \frac{\partial u}{\partial n}=0 & x \in \partial \Omega\end{cases}
$$

Let $r N=\lambda_{m}(m \in \mathbb{N})$ be an eigenvalue of the Laplacian operator $-\Delta$ with zero flux on the boundary and $u_{m}$ be the eigenfunction associated to $\lambda_{m}$. Then

$$
p=N\left(\frac{1}{2}+\frac{1}{2}\left(1-4 \frac{u_{m}}{C N}\right)^{1 / 2}\right)
$$

is a solution to the problem for any $C \geqslant C^{*}:=(4 / N) \max _{x \in \Omega}\left\{\left|u_{m}\right|\right\}$. Notice that $p>0$.

\section{EXISTENCE OF SOLUTION (PROOF OF THEOREM 1.2)}

We introduce the function

$$
f(w)=\exp \left[\int_{0}^{w} \chi(s) \mathrm{d} s\right]
$$

and the unknown $q$ defined by

$$
p=f(w) q
$$

In terms of $q$ and $w$ the system (2)-(3) becomes

$$
\begin{aligned}
\mathscr{L} q & \equiv \frac{\partial q}{\partial t}-\Delta q-\chi(w) \nabla w \cdot \nabla q \\
& =-q \chi(w) h(q f(w), w)+r q(N-q f(w)) \quad x \in \Omega \quad t>0 \\
\frac{\partial w}{\partial t} & =h(q f(w), w) \quad x \in \Omega \quad t>0
\end{aligned}
$$

and

$$
\frac{\partial q}{\partial n}=0 \quad x \in \partial \Omega \quad t>0
$$

The initial conditions (4) become

$$
q(x, 0)=q_{0}(x)=\frac{p_{0}(x)}{f\left(w_{0}(x)\right)} \quad w(0, x)=w_{0}(x)
$$


Notice that

$$
f(w) \cdot \mathscr{L} q=f(w) \frac{\partial q}{\partial t}-\operatorname{div}\{f(w) \nabla q\}
$$

Denote the right hand side of (29) by $g(q, w)$.

Definition 3.1

$q \in L^{2}\left(0, T: H^{2}(\Omega)\right) \cap H^{1}\left(0, T: L^{2}(\Omega)\right)$ and $w \in L^{\infty}\left(0, T: L^{2}(\Omega)\right)$ is a weak solution to (29) $-(32)$ if

$$
\iint_{\Omega_{T}} f(w) q_{t} \eta \mathrm{d} \sigma \mathrm{d} t+\iint_{\Omega_{T}} f(w) \nabla q \nabla \eta \mathrm{d} \sigma \mathrm{d} t=\iint_{\Omega_{T}} f(w) g(q, w) \eta \mathrm{d} \sigma \mathrm{d} t
$$

for any $\eta \in L^{2}\left(0, T: H^{1}(\Omega)\right)$ and

$$
w_{t}=h(q f(w), w) \quad \text { a.e. } \quad 0<t<T \quad x \in \Omega
$$

In order to establish the existence of a global solution, we consider the sequence $q_{i}$ defined as the unique solution to (29), (31) where $w=w_{i}$ and $w_{i}$ satisfies

$$
\frac{\partial w_{i}}{\partial t}=h\left(q_{i-1} f\left(w_{i}\right), w_{i}\right) \quad x \in \Omega \quad 0<t<T, \quad w(x, 0)=w_{0}(x)
$$

Let us denote $q_{i}$ by $q$ and $w_{i}$ by $w$, then

Lemma 3.1

$q \geqslant 0$ and $0 \leqslant w \leqslant \bar{w}$.

Proof

By the maximum principle we get $q \geqslant 0$. Since $\partial h / \partial q=f(w) \partial h / \partial p>0$, we have $h(0, w) \leqslant w_{t} \leqslant$ $h(\bar{q}, w)$. By (7) and (6) we conclude the result.

Lemma 3.2

$$
q(x, t) \leqslant \bar{q}
$$

Proof

Let us take $f(w)(q-\bar{q})_{+}$as test function in (29), then

$$
\begin{aligned}
& \frac{1}{2} \int_{\Omega} f(w)(q-\bar{q})_{+}^{2} \mathrm{~d} \sigma \mathrm{d} t+\frac{1}{2} \iint_{\Omega_{T}} f^{\prime}(w) w_{t}(q-\bar{q})_{+}^{2} \mathrm{~d} \sigma \mathrm{d} t+\iint_{\Omega_{T}} f(w)\left(\nabla(q-\bar{q})_{+}\right)^{2} \mathrm{~d} \sigma \mathrm{d} t \\
& \quad=\iint_{\Omega_{T}} f(w) g(q, w)(q-\bar{q})_{+}^{2} \mathrm{~d} \sigma \mathrm{d} t+\int_{\Omega} f\left(w_{0}\right)\left(q_{0}-\bar{q}\right)_{+}^{2} \mathrm{~d} \sigma \mathrm{d} t
\end{aligned}
$$

Since $g(q, w)(q-\bar{q})_{+} \leqslant 0$ provided that $0 \leqslant w \leqslant \bar{w}$ we get

$$
\int_{\Omega} f(w)(q-\bar{q})_{+}^{2} \mathrm{~d} \sigma \mathrm{d} t \leqslant C \iint_{\Omega_{T}}(q-\bar{q})_{+}^{2} \mathrm{~d} \sigma \mathrm{d} t
$$

By Gronwall's Lemma we prove the lemma. 
Provided that $0 \leqslant q<\bar{q}$ and $0 \leqslant w<\bar{w}$, we show the following a priori estimates:

Lemma 3.3

$$
\int_{\Omega} q^{2} \mathrm{~d} \sigma+\iint_{\Omega_{T}}|\nabla q|^{2} \mathrm{~d} \sigma \mathrm{d} t+\int_{\Omega}|\nabla w|^{2} \mathrm{~d} \sigma \leqslant C_{0}(T+1)
$$

Proof

From (1) we have

$$
\nabla w_{t}=f(w) h_{p} \nabla q+\left(h_{p} f(w) q \chi(w)+h_{w}\right) \nabla w
$$

Taking the scalar product with $\nabla w$, integrating over $\Omega_{T}$ and using (8), (9) we find that

$$
\int_{\Omega}|\nabla w|^{2} \mathrm{~d} \sigma \leqslant C_{1} \iint_{\Omega_{T}}|\nabla q|^{2} \mathrm{~d} \sigma \mathrm{d} t+\int_{\Omega}\left|\nabla w_{0}\right|^{2} \mathrm{~d} \sigma
$$

Taking $f(w) q$ as test function in (29) and integrating by parts, it follows

$$
\begin{aligned}
\frac{\mathrm{d}}{\mathrm{d} t} & \frac{1}{2} \int_{\Omega} q^{2} f(w) \mathrm{d} \sigma+\int_{\Omega} f(w)|\nabla q|^{2} \mathrm{~d} \sigma \\
& =-\frac{1}{2} \int_{\Omega} q^{2} f(w) \chi(w) h(q f(w), w) \mathrm{d} \sigma+\int_{\Omega} q^{2} f(w)(N-q f(w)) \mathrm{d} \sigma \leqslant C_{2}
\end{aligned}
$$

and then

$$
\frac{1}{2} \int_{\Omega} q^{2} f(w) \mathrm{d} \sigma+\iint_{\Omega_{T}} f(w)|\nabla q|^{2} \mathrm{~d} \sigma \mathrm{d} t \leqslant C_{2} T+\frac{1}{2} \int_{\Omega} q_{0}^{2} f\left(w_{0}\right) \mathrm{d} \sigma
$$

Since $w \geqslant 0$, by (27) and (10) we get

$$
\int_{\Omega} q^{2} \mathrm{~d} \sigma+\iint_{\Omega_{T}}|\nabla q|^{2} \mathrm{~d} \sigma \mathrm{d} t \leqslant C_{2}(T+1)
$$

Substituting (37) into (36) we prove the lemma.

Lemma 3.4

$$
\iint_{\Omega_{T}} q_{t}^{2} \mathrm{~d} \sigma \mathrm{d} t+\int_{\Omega}|\nabla q|^{2} \mathrm{~d} \sigma \leqslant C_{3}(T+1)
$$

Proof

Let us take $f(w) q_{t}$ as test function in (29), we obtain

$$
\int_{\Omega} f(w)\left|q_{t}\right|^{2} \mathrm{~d} \sigma-\int_{\Omega}(\operatorname{div} f(w) \nabla q) q_{t} \mathrm{~d} \sigma=\int_{\Omega} f(w) g(q, w) q_{t} \mathrm{~d} \sigma
$$


Since

$$
\begin{aligned}
& -\int_{\Omega}(\operatorname{div} f(w) \nabla q) q_{t} \mathrm{~d} \sigma=\frac{1}{2} \int_{\Omega} f(w) \frac{\partial}{\partial t}|\nabla q|^{2} \mathrm{~d} \sigma \\
& =\frac{\partial}{\partial t} \frac{1}{2} \int_{\Omega} f(w)|\nabla q|^{2} \mathrm{~d} \sigma-\frac{1}{2} \int_{\Omega} f(w) \chi(w) w_{t}|\nabla q|^{2} \mathrm{~d} \sigma \\
& =\frac{\partial}{\partial t} \frac{1}{2} \int_{\Omega} f(w)|\nabla q|^{2} \mathrm{~d} \sigma-\frac{1}{2} \int_{\Omega} f(w) \chi(w) h(q f(w), w)|\nabla q|^{2} \mathrm{~d} \sigma
\end{aligned}
$$

and

$$
\int_{\Omega} f(w) g(q, w) q_{t} \mathrm{~d} \sigma \leqslant \frac{1}{2} \int_{\Omega} f(w)\left|q_{t}\right|^{2} \mathrm{~d} \sigma+\frac{1}{2} \int_{\Omega} f(w) g^{2}(q, w) \mathrm{d} \sigma
$$

then, substituting it into (38) we find

$$
\int_{\Omega} f(w)\left|q_{t}\right|^{2} \mathrm{~d} \sigma+\frac{\partial}{\partial t} \frac{1}{2} \int_{\Omega} f(w)|\nabla q|^{2} \mathrm{~d} \sigma-\frac{1}{2} \int_{\Omega} f(w) \chi(w) h(q f(w), w)|\nabla q|^{2} \mathrm{~d} \sigma \leqslant C
$$

Integrate with respect to time to get

$$
\iint_{\Omega_{T}} f(w)\left|q_{t}\right|^{2} \mathrm{~d} \sigma+\int_{\Omega} f(w)|\nabla q|^{2} \mathrm{~d} \sigma \leqslant C_{5}\left[\iint_{\Omega_{T}}|\nabla q|^{2} \mathrm{~d} \sigma \mathrm{d} t+\int_{\Omega} f\left(w_{0}\right)\left|\nabla q_{0}\right|^{2} \mathrm{~d} \sigma+T\right]
$$

By selection of $f$ and Lemma 3.3 we obtain the desired result.

Lemma 3.5

$q$ belongs to $L^{2}\left(0, T: H^{2}(\Omega)\right)$.

Proof

By the previous lemma we know that $(1 / f(w)) \operatorname{div}\{f(w) \nabla q\}$ belongs to $L^{2}\left(\Omega_{T}\right)$ i.e.

$$
\begin{aligned}
& \iint_{\Omega_{T}} \frac{1}{f^{2}(w)}(\operatorname{div}\{f(w) \nabla q\})^{2} \mathrm{~d} \sigma \mathrm{d} t \\
& =\iint_{\Omega_{T}}\left(\frac{1}{f^{2}(w)}(\nabla f(w) \cdot \nabla q)^{2}+(\Delta q)^{2}\right) \mathrm{d} \sigma \mathrm{d} t \\
& \quad+\iint_{\Omega_{T}} \frac{2}{f(w)}(\Delta q)(\nabla f(w) \cdot \nabla q) \mathrm{d} \sigma \mathrm{d} t \leqslant C_{3}(T+1)
\end{aligned}
$$

Then

$$
\iint_{\Omega_{T}} \frac{1}{f(w)}(\Delta q)(\nabla f(w) \cdot \nabla q) \mathrm{d} \sigma \mathrm{d} t \leqslant \frac{C_{3}}{2}(T+1)
$$


Let us take $-\Delta q$ as test function in (29) then:

$$
\begin{aligned}
& \iint_{\Omega_{T}}(-\Delta q) q_{t} \mathrm{~d} \sigma \mathrm{d} t+\iint_{\Omega_{T}}(\Delta q)^{2} \mathrm{~d} \sigma \mathrm{d} t+\iint_{\Omega_{T}} \frac{1}{f(w)}(\Delta q)(\nabla f(w) \cdot \nabla q) \mathrm{d} \sigma \mathrm{d} t \\
& =\iint_{\Omega_{T}} g(q, w)(-\Delta q) \mathrm{d} \sigma \mathrm{d} t \leqslant \frac{T|\Omega|}{2}(\max \{g(q, w)\})^{2}+\frac{1}{2} \iint_{\Omega_{T}}(\Delta q)^{2} \mathrm{~d} \sigma \mathrm{d} t
\end{aligned}
$$

Then, integrating by parts on the left hand side of the equation and as a result of (39) we conclude

$$
\left.\frac{1}{2} \int_{\Omega}|\nabla q|^{2} \mathrm{~d} \sigma\right|_{0} ^{T}+\frac{1}{2} \iint_{\Omega_{T}}(\Delta q)^{2} \mathrm{~d} \sigma \mathrm{d} t \leqslant C_{4}(T+1)
$$

which proves the lemma.

Lemma 3.6

$\int_{\Omega}|\nabla w|^{4} \mathrm{~d} \sigma \leqslant C_{5}(T+1)$.

Proof

As a consequence of Lemma 3.5 and (29) we obtain that $\nabla q \cdot \nabla w$ belongs to $L^{2}\left(\Omega_{T}\right)$ (using (10) and (7)). Multiplying by $|\nabla w|^{2} \nabla w$ in (35) and integrating over $\Omega_{T}$ we conclude the claim of the lemma.

Lemma 3.7

$w \in L^{\infty}\left(0, T: H^{2}(\Omega)\right)$.

Proof

By (30) we know that

$$
\Delta w_{t}=h_{q} \Delta q+h_{q q}|\nabla q|^{2}+2 h_{q w} \nabla q \cdot \nabla w h_{w w}|\nabla w|^{2}+h_{w} \Delta w
$$

Multiplying by $\Delta w$ in (40) and applying Hölder inequality, one gets in view of (9)

$$
\frac{\partial}{\partial t} \frac{1}{2}|\Delta w|^{2} \leqslant \frac{h_{q}^{2}}{\left|h_{w}\right|}|\Delta q|^{2}+\frac{h_{q q}^{2}}{\left|h_{w}\right|}|\nabla q|^{4}+4 \frac{h_{q w}^{2}}{\left|h_{w}\right|}|\nabla q|^{2}|\nabla w|^{2}+\frac{h_{w w}^{2}}{\left|h_{w}\right|}|\nabla w|^{4}
$$

Integrating over $\Omega_{T}$ we conclude the lemma.

Proof of Theorem 1.2

Let us consider the sequence $\left\{p_{i}=q_{i} f\left(w_{i}\right)\right\}_{i=1, \infty}$. From Lemmas 3.1, 3.2 and 3.4

$$
p_{i} \text { are uniformly bounded in } H^{1}\left(0, T: L^{2}(\Omega)\right) \cap L^{\infty}\left(\Omega_{T}\right)
$$

and by Lemmas 3.5 and 3.6

$$
p_{i} \text { are uniformly bounded in } L^{2}\left(0, T: H^{2}(\Omega)\right)
$$

Let us consider

$$
\tilde{p}=p_{i}-p_{j}, \quad \tilde{p}_{-1}=p_{i-1}-p_{j-1}, \quad \gamma(w)=\int_{0}^{w} \chi(s) \mathrm{d} s \quad \text { and } \quad \tilde{\gamma}=\gamma\left(w_{i}\right)-\gamma\left(w_{j}\right)
$$


Then $(\tilde{p}, \tilde{\gamma})$ satisfy

$$
\begin{gathered}
\frac{\partial \tilde{p}}{\partial t}-\Delta \tilde{p}+\operatorname{div}\left\{\tilde{p} \nabla \gamma\left(w_{i}\right)\right\}+\operatorname{div}\left\{p_{j} \nabla \tilde{\gamma}\right\}=\tilde{p}\left(1-\left(p_{i}+p_{j}\right)\right) \text { in } \Omega_{T} \\
\frac{\partial \tilde{\gamma}}{\partial t}=\chi\left(w_{i}\right) h\left(p_{i-1}, w_{i}\right)-\chi\left(w_{j}\right) h\left(p_{j-1}, w_{j}\right) \quad \text { in } \Omega_{T}
\end{gathered}
$$

Take $f\left(w_{i}\right) \tilde{p}$ as test function in (43), then

$$
\begin{aligned}
& \left.\frac{1}{2} \int_{\Omega} f\left(w_{i}\right) \tilde{p}^{2} \mathrm{~d} \sigma\right|_{T}-\frac{1}{2} \iint_{\Omega_{T}} f^{\prime}\left(w_{i}\right) \frac{\partial w_{i}}{\partial t} \tilde{p}^{2} \mathrm{~d} \sigma \mathrm{d} t+\iint_{\Omega_{T}} f\left(w_{i}\right)|\nabla \tilde{p}|^{2} \mathrm{~d} \sigma \mathrm{d} t \\
& \quad+\iint_{\Omega_{T}} \tilde{p} \nabla \tilde{p} \nabla f\left(w_{i}\right) \mathrm{d} \sigma \mathrm{d} t-\iint_{\Omega_{T}} \tilde{p}^{2} \nabla \gamma\left(w_{i}\right) \nabla f\left(w_{i}\right) \mathrm{d} \sigma \mathrm{d} t \\
& \quad-\iint_{\Omega_{T}} f\left(w_{i}\right) \tilde{p} \nabla \gamma\left(w_{i}\right) \nabla \tilde{p} \mathrm{~d} \sigma \mathrm{d} t-\iint_{\Omega_{T}} f\left(w_{i}\right) p_{j} \nabla \tilde{\gamma} \nabla \tilde{p} \mathrm{~d} \sigma \mathrm{d} t \\
& \quad-\iint_{\Omega_{T}} p_{j} \tilde{p} \nabla \tilde{\gamma} \nabla f\left(w_{i}\right) \mathrm{d} \sigma \mathrm{d} t=\iint_{\Omega_{T}} \tilde{p} f\left(w_{i}\right)\left(\hat{g}\left(p_{i}, w_{i}\right)-g\left(p_{i}, w_{i}\right)\right) \mathrm{d} \sigma \mathrm{d} t
\end{aligned}
$$

Since $\left|(\partial / \partial t) w_{i}\right| \leqslant C, 1 \leqslant f\left(w_{i}\right) \leqslant C$ and $\nabla f\left(w_{i}\right)=f\left(w_{i}\right) \nabla \gamma\left(w_{i}\right)$, we obtain

$$
\begin{aligned}
& \left.\frac{1}{2} \int_{\Omega} \tilde{p}^{2} \mathrm{~d} \sigma\right|_{T}+\iint_{\Omega_{T}}|\nabla \tilde{p}|^{2} \mathrm{~d} \sigma \mathrm{d} t \\
& \quad \leqslant \bar{p} \iint_{\Omega_{T}}|\nabla \tilde{\gamma}||\nabla \tilde{p}| \mathrm{d} \sigma \mathrm{d} t+\iint_{\Omega_{T}}|\tilde{p}||\nabla \tilde{\gamma}|\left|\nabla \gamma\left(w_{i}\right)\right| \mathrm{d} \sigma \mathrm{d} t+k_{0} \iint_{\Omega_{T}} \tilde{p}^{2}+\tilde{\gamma}^{2} \mathrm{~d} \sigma \mathrm{d} t
\end{aligned}
$$

Let us consider the integral

$$
\bar{p} \iint_{\Omega_{T}}|\nabla \tilde{\gamma}||\nabla \tilde{p}| \mathrm{d} \sigma \leqslant \bar{p}^{2}\|\nabla \tilde{\gamma}\|_{L^{2}\left(\Omega_{T}\right)}^{2}+\frac{1}{4}\|\nabla \tilde{p}\|_{L^{2}\left(\Omega_{T}\right)}^{2}
$$

and

$$
\begin{gathered}
\iint_{\Omega_{T}}|\tilde{p}|\left|\nabla \gamma\left(w_{i}\right)\right||\nabla \tilde{\gamma}| \mathrm{d} \sigma \mathrm{d} t \leqslant\|\tilde{p}\|_{L^{2}\left(0, T: L^{4}(\Omega)\right)}\left\|\nabla \gamma\left(w_{i}\right)\right\|_{L^{\infty}\left(0, T: L^{4}(\Omega)\right)}\|\nabla \tilde{\gamma}\|_{L^{2}\left(0, T: L^{2}(\Omega)\right)} \\
\quad \leqslant k_{1}\|\tilde{p}\|_{L^{2}\left(0, T: H^{1}(\Omega)\right)}\|\nabla \tilde{\gamma}\|_{L^{2}\left(0, T: L^{2}(\Omega)\right)} \leqslant \frac{1}{4}\|\tilde{p}\|_{L^{2}\left(0, T: H^{1}(\Omega)\right)}^{2}+k_{1}^{2}\|\nabla \tilde{\gamma}\|_{L^{2}\left(0, T: L^{2}(\Omega)\right)}^{2}
\end{gathered}
$$

Substituting it in (45) we get

$$
\left.\frac{1}{2} \int_{\Omega} \tilde{p}^{2} \mathrm{~d} \sigma\right|_{T}+\frac{1}{2} \iint_{\Omega_{T}}|\nabla \tilde{p}|^{2} \mathrm{~d} \sigma \mathrm{d} t \leqslant k_{2}\left(\iint_{\Omega_{T}} \tilde{p}^{2}+\tilde{\gamma}^{2} \mathrm{~d} \sigma \mathrm{d} t+\iint_{\Omega_{T}}|\nabla \tilde{\gamma}|^{2} \mathrm{~d} \sigma \mathrm{d} t\right)
$$

Notice that, as below, if $t \leqslant T$ we obtain

$$
\left.\frac{1}{2} \int_{\Omega} \tilde{p}^{2} \mathrm{~d} \sigma\right|_{t} \leqslant k_{2}\left(\int_{0}^{T} \int_{\Omega} \tilde{p}^{2}+\tilde{\gamma}^{2} \mathrm{~d} \sigma \mathrm{d} t+\int_{0}^{T} \int_{\Omega}|\nabla \tilde{\gamma}|^{2} \mathrm{~d} \sigma \mathrm{d} t\right)
$$


and integrating again in time we get

$$
\frac{1}{2} \iint_{\Omega_{T}} \tilde{p}^{2} \mathrm{~d} \sigma \mathrm{d} t \leqslant T k_{2}\left(\iint_{\Omega_{T}} \tilde{p}^{2}+\tilde{\gamma}^{2} \mathrm{~d} \sigma \mathrm{d} t+\iint_{\Omega_{T}}|\nabla \tilde{\gamma}|^{2} \mathrm{~d} \sigma \mathrm{d} t\right)
$$

Taking $T<1 / 4 k_{2}$ it results

$$
\iint_{\Omega_{T}} \tilde{p}^{2} \mathrm{~d} \sigma \mathrm{d} t \leqslant 4 T k_{2}\left(\iint_{\Omega_{T}} \tilde{\gamma}^{2} \mathrm{~d} \sigma \mathrm{d} t+\iint_{\Omega_{T}}|\nabla \tilde{\gamma}|^{2} \mathrm{~d} \sigma \mathrm{d} t\right)
$$

Then, by (46) and (47) $\tilde{p}$ satisfies

$$
\iint_{\Omega_{T}} \tilde{p}^{2} \mathrm{~d} \sigma \mathrm{d} t+\iint_{\Omega_{T}}|\nabla \tilde{p}|^{2} \mathrm{~d} \sigma \mathrm{d} t \leqslant\left(4 T k_{2}+2 k_{2}\left(1+4 T k_{2}\right)\right) \iint_{\Omega_{T}}\left(\tilde{\gamma}^{2}+|\nabla \tilde{\gamma}|^{2}\right) \mathrm{d} \sigma \mathrm{d} t
$$

On the other hand, $\tilde{\gamma}$ satisfies:

$$
\frac{\partial}{\partial t} \tilde{\gamma}=\left.h_{p}\right|_{\hat{p}, \hat{w}} \tilde{p}_{-1}+\left.h_{\gamma}\right|_{\hat{p}, \hat{w} \tilde{\gamma}}
$$

where $p_{i} \leqslant \hat{p} \leqslant p_{j}$ or $p_{i} \geqslant \hat{p} \geqslant p_{j}$ and $w_{i} \leqslant \hat{w} \leqslant w_{j}$ or $w_{i} \geqslant \hat{w} \geqslant w_{j}$ and $h_{\gamma}=h_{w} / \gamma^{\prime}<0$.

Multiply by $\tilde{\gamma}$, integrate and apply Hölder inequality to get

$$
\left.\int_{\Omega} \tilde{\gamma}^{2} \mathrm{~d} \sigma\right|_{t} \leqslant k_{3} \iint_{\Omega_{T}} \tilde{p}_{-1}^{2} \mathrm{~d} \sigma \mathrm{d} t+\frac{1}{2} \iint_{\Omega_{T}} h_{\gamma} \tilde{\gamma}^{2} \mathrm{~d} \sigma \mathrm{d} t
$$

i.e.

$$
\iint_{\Omega_{T}} \tilde{\gamma}^{2} \mathrm{~d} \sigma \mathrm{d} t \leqslant k_{3} T \iint_{\Omega_{T}} \tilde{p}_{-1}^{2} \mathrm{~d} \sigma \mathrm{d} t+\frac{T}{2} \iint_{\Omega_{T}} h_{\gamma} \tilde{\gamma}^{2} \mathrm{~d} \sigma \mathrm{d} t
$$

Since $\nabla \tilde{\gamma}$ satisfies

$$
\begin{aligned}
\frac{\partial}{\partial t} \nabla \tilde{\gamma}= & \left.h_{p}\right|_{\hat{p}, \hat{\gamma}} \nabla \tilde{p}_{-1}+\left.h_{p p}\right|_{\hat{p}, \hat{\gamma}} \tilde{p}_{-1} \nabla \hat{p} \\
& +\left.h_{p \gamma}\right|_{\hat{p}, \hat{\gamma}}\left(\tilde{p}_{-1} \nabla \hat{\gamma}+\tilde{\gamma} \nabla \hat{p}\right)+\left.h_{\gamma}\right|_{\hat{p}, \hat{\gamma}} \nabla \tilde{\gamma}+\left.h_{\gamma \gamma}\right|_{\hat{p}, \hat{\gamma}} \tilde{\gamma} \nabla \hat{\gamma}
\end{aligned}
$$

taking $\nabla \tilde{\gamma}$ as test function and by Hölder inequality we get

$$
\left.\int_{\Omega}|\nabla \tilde{\gamma}|^{2} \mathrm{~d} \sigma \mathrm{d} t\right|_{t} \leqslant k_{4}\left(\left\|\tilde{p}_{-1}\right\|_{L^{2}\left(0, T: H^{1}(\Omega)\right\}}^{2}+\|\tilde{\gamma}\|_{L^{2}\left(0, T: H^{1}(\Omega)\right\rangle}^{2}\right)
$$

and

$$
\iint_{\Omega_{T}}|\nabla \tilde{\gamma}|^{2} \mathrm{~d} \sigma \mathrm{d} t \leqslant T k_{4}\left(\left\|\tilde{p}_{-1}\right\|_{L^{2}\left(0, T: H^{1}(\Omega)\right)}^{2}+\|\tilde{\gamma}\|_{L^{2}\left(0, T: H^{1}(\Omega)\right)}^{2}\right)
$$

Adding (49) to (51) we get

$$
\left\|\tilde{\gamma}^{2}\right\|_{L^{2}\left(0, T: H^{1}(\Omega)\right)} \leqslant T k_{5}\left(\left\|\tilde{p}_{-1}\right\|_{L^{2}\left(0, T: H^{1}(\Omega)\right)}^{2}+\|\tilde{\gamma}\|_{L^{2}\left(0, T: H^{1}(\Omega)\right)}^{2}\right)
$$

Taking $T \leqslant 1 / 2 k_{5}$ it results

$$
\iint_{\Omega_{T}} \tilde{\gamma}^{2} \mathrm{~d} \sigma \mathrm{d} t+\iint_{\Omega_{T}}|\nabla \tilde{\gamma}|^{2} \mathrm{~d} \sigma \mathrm{d} t \leqslant 2 T k_{5}\left\|\tilde{p}_{-1}\right\|_{L^{2}\left(0, T: H^{1}(\Omega)\right)}^{2}
$$


Substitute (52) into (48)

$$
\iint_{\Omega_{T}} \tilde{p}^{2} \mathrm{~d} \sigma \mathrm{d} t+\iint_{\Omega_{T}}|\nabla \tilde{p}|^{2} \mathrm{~d} \sigma \mathrm{d} t \leqslant\left(4 T k_{2}+2 k_{2}\left(1+4 T k_{2}\right)\right) 2 T k_{5}\left\|\tilde{p}_{-1}\right\|_{L^{2}\left(0, T: H^{1}(\Omega)\right)}^{2}
$$

Choose $T$ small enough, then

$$
\|\tilde{p}\|_{L^{2}\left(0, T: H^{1}(\Omega)\right)} \leqslant \frac{1}{2}\left\|\tilde{p}_{-1}\right\|_{L^{2}\left(0, T: H^{1}(\Omega)\right)}
$$

Then $p_{i}$ is a Cauchy sequence satisfying $p_{i} \rightarrow p$ in $L^{2}\left(0, T: H^{1}(\Omega)\right)$. By (52) we get the same result for $w_{i}$ and $\gamma\left(w_{i}\right)$. Since $\left\{p_{i}, \gamma\left(w_{i}\right)\right\}_{i=1}^{\infty}$ are uniformly bounded in

$$
\left[L^{2}\left(0, T: H^{2}(\Omega)\right) \cap H^{1}\left(0, T: L^{2}(\Omega)\right) \cap L^{\infty}\left(\Omega_{T}\right)\right]^{2}
$$

there exists a subsequence $\left(p_{i j}, \gamma\left(w_{i j}\right)\right)$ such that $\left(p_{i j}, \gamma\left(w_{i j}\right)\right) \rightarrow(p, \gamma(w))$ in $\left[L^{2}\left(0, T: W^{1, s}(\Omega)\right)\right]^{2}$ for any $s \leqslant \infty$ if $n=1, s<\infty$ if $n=2$ and $s<2 n /(n-2)$ if $n=3$ and weakly in $\left[H^{1}\left(0, T: L^{2}(\Omega)\right)\right]^{2}$. By $(50)$ and a priori estimates, we obtain that $\gamma\left(w_{i j}\right) \rightarrow \gamma(w)$ in $L^{r}\left(0, T: H^{1}(\Omega)\right)$ for arbitrary $r<\infty$.

Taking limits in the weak formulation

$$
\begin{gathered}
\iint_{\Omega_{T}} \frac{\partial}{\partial t} p_{i j} \eta \mathrm{d} \sigma \mathrm{d} t+\iint_{\Omega_{T}} \nabla p_{i j} \cdot \nabla \eta \mathrm{d} \sigma \mathrm{d} t \\
=\iint_{\Omega_{T}} p_{i j} \nabla \gamma\left(w_{i j}\right) \cdot \nabla \eta \mathrm{d} \sigma \mathrm{d} t+\iint_{\Omega_{T}} \hat{g}\left(p_{i j}, w_{i j}\right) \eta \mathrm{d} \sigma \mathrm{d} t \\
\frac{\partial}{\partial t} w_{i j}=h\left(p_{i j}, w_{i j}\right)
\end{gathered}
$$

we get the existence of weak solutions for small $T$. Repeating the process, starting now from $T$, we conclude the existence of solutions for arbitrary $T>0$ by lemmata 3.1-3.7.

Remark 3.1

Uniqueness of solutions is a consequence of (53).

\section{ON STABILITY (PROOF OF THEOREM 1.3)}

Taking $p-N$ as test function in (2) we get

$$
\begin{aligned}
& \left.\frac{1}{2} \int_{\Omega}(p-N)^{2} \mathrm{~d} \sigma\right|_{0} ^{T}+\iint_{\Omega_{T}}|\nabla p|^{2} \mathrm{~d} \sigma \mathrm{d} t \\
& \quad=\iint_{\Omega_{T}} p \chi(w) \nabla p \cdot \nabla w \mathrm{~d} \sigma \mathrm{d} t+r \iint_{\Omega_{T}} p(p-N)^{2} \mathrm{~d} \sigma \mathrm{d} t
\end{aligned}
$$

Since $\nabla w_{t}=h_{p} \nabla p+h_{w} \nabla w$, taking scalar product with $\gamma \nabla w$ (for a positive constant $\gamma$ ) and integrating over $\Omega_{T}$ (as in Reference [5]) we find that

$$
\left.\frac{\gamma}{2} \int_{\Omega}|\nabla w|^{2} \mathrm{~d} \sigma\right|_{0} ^{T}=\gamma \iint_{\Omega_{T}} h_{w}|\nabla w|^{2} \mathrm{~d} \sigma \mathrm{d} t+\gamma \iint_{\Omega_{T}} h_{p} \nabla p \cdot \nabla w \mathrm{~d} \sigma \mathrm{d} t
$$


Adding both expressions one concludes

$$
\begin{aligned}
& \left.\frac{1}{2} \int_{\Omega}(p-N)^{2} \mathrm{~d} \sigma\right|_{T}+\left.\left.\iint_{\Omega_{T}}\left|\nabla p^{2} \mathrm{~d} \sigma \mathrm{d} t+\frac{\gamma}{2} \int_{\Omega}\right| \nabla w\right|^{2} \mathrm{~d} \sigma\right|_{T}-\gamma \iint_{\Omega_{T}} h_{w}|\nabla w|^{2} \mathrm{~d} \sigma \mathrm{d} t \\
& \quad=r \iint_{\Omega_{T}} p(p-N)^{2} \mathrm{~d} \sigma \mathrm{d} t+\iint_{\Omega_{T}}\left(p \chi(w)+\gamma h_{p}\right) \nabla p \cdot \nabla w \mathrm{~d} \sigma \mathrm{d} t+O(1)
\end{aligned}
$$

By Schwarz's inequality, the last integral is bounded by

$$
(1-\delta) \iint_{\Omega_{T}}|\nabla p|^{2} \mathrm{~d} \sigma \mathrm{d} t+\frac{1}{4(1-\delta)} \iint_{\Omega_{T}}\left(p \chi(w)+\gamma h_{p}\right)^{2}|\nabla w|^{2} \mathrm{~d} \sigma \mathrm{d} t
$$

for any $1>\delta>0$ and $\gamma>0$.

Consider the quadratic equation in $\gamma$ :

$$
\left(\gamma h_{p}+p \chi(w)\right)^{2}+4 \gamma h_{w}=0
$$

and denote its two roots by

$$
\gamma_{1,2}(p, w)=\frac{2}{h_{p}^{2}}\left\{\left(-2 h_{w}-h_{p} p \chi(w)\right) \pm\left[\left(2 h_{w}+h_{p} p \chi(w)\right)^{2}-(p \chi(w))^{2} h_{p}{ }^{2}\right]^{1 / 2}\right\}
$$

By assumption (9) there exists $\gamma>0$ such that

$$
\left(\gamma h_{p}+p \chi(w)\right)^{2}<4 \gamma h_{w}
$$

Therefore

$$
\begin{aligned}
& \left.\int_{\Omega}(p-N)^{2} \mathrm{~d} \sigma\right|_{T}+r \iint_{\Omega_{T}} p(p-N)^{2} \mathrm{~d} \sigma \mathrm{d} t+\left.\int_{\Omega}|\nabla w|^{2} \mathrm{~d} \sigma\right|_{T} \\
& +\delta \iint_{\Omega_{T}}|\nabla p|^{2} \mathrm{~d} \sigma \mathrm{d} t+\varepsilon \iint_{\Omega_{T}}|\nabla w|^{2} \mathrm{~d} \sigma \mathrm{d} t \leqslant O(1)
\end{aligned}
$$

which implies

$$
\iint_{\Omega_{\infty}}|\nabla p|^{2} \mathrm{~d} \sigma \mathrm{d} t+\iint_{\Omega_{\infty}}|\nabla w|^{2} \mathrm{~d} \sigma \mathrm{d} t \leqslant C
$$

Integrating the equation we get

$$
\frac{\partial}{\partial t} \int_{\Omega} p \mathrm{~d} \sigma=r\left(N \int_{\Omega} p \mathrm{~d} \sigma-\int_{\Omega} p^{2} \mathrm{~d} \sigma\right)
$$

which implies that $p=0$ is unstable, and we deduce that

$$
p \rightarrow N \quad \text { and } \quad w \rightarrow \Psi(N) \text { in } L^{s}(\Omega)
$$

where $s=\infty$ if $n=1, s<\infty$ if $n=2$ and $s<6$ if $n=3$. 


\section{REFERENCES}

1. Keller EF, Segel LA. Initiation of slime mold aggregation viewed as an instability. Journal of Theoretical Biology $1970 ; 26: 399-415$.

2. Keller EF, Segel LA. A model for chemotaxis. Journal of Theoretical Biology 1971; 30:225-234.

3. Corrias L, Perthame B, Zaag H. A chemotaxis model motivated by angiogenesis. Royal Mathematica Academiae Scientasium Paris 2003; 336:141-146.

4. Fontelos MA, Friedman A, Hu B. Mathematical analysis of a model for the initiation of angiogenesis. SIAM Journal on Mathematical Analysis 2002; 33:1330-1355.

5. Friedman A, Tello JI. Stability of solutions of chemotaxis equations in reinforced random walks. Journal of Mathematical Analysis and Applications 2002; 272:138-163.

6. Herrero MA, Velázquez JJL. A blow-up mechanism for a chemotaxis model. Ann. Scuola. Norm. Sup. Pisa. Cl. Sci. 1997; 24(4):633-683.

7. Jäger W, Luckhaus S. On explosions of solutions to a system of partial differential equations modeling chemotaxis. Transactions of the American Mathematical Society 1992; 329:819-824.

8. Othmer HG, Stevens A. Aggregation, blowup, and collapse: the ABC's of taxis in reinforced random walks. SIAM Journal on Applied Mathematics 1997 57:1044-1081.

9. Levine HA, Sleeman BP. A system of reaction diffusion equations arising in the theory of reinforced random walks. SIAM Journal on Applied Mathematics 1997; 57:683-730.

10. Anderson ARA, Chaplain MAJ. Continuous and discrete mathematical models of tumor-induced angiogenesis. Bulletin of Mathematical Biology 1998; 60:857-899.

11. Levine HA, Sleeman BP, Nilsen-Hamilton N. A mathematical modeling for the roles of pericytes and macrophages in the initiation of angiogenesis $\mathrm{I}$. The role of protease inhibitors in preventing angiogenesis. Mathematical Biosciences 2000; 168:75-115.

12. Myerscough MR, Maini PK, Painter J. Pattern formation in a generalized chemotactic model. Bulletin of Mathematical Biology 1998; 60:1-26. 